Research Article

\title{
Development of a Chemiluminescence Immunoassay for Quantification of 25-Hydroxyvitamin D in Human Serum
}

\author{
Shuang Han, ${ }^{1,2}$ Wuxian Qiu, ${ }^{3}$ Junlan Zhang, ${ }^{1}$ Zhonghu Bai $\mathbb{D}^{1,2,4}$ and Xiao Tong ${ }^{3}{ }^{3}$ \\ ${ }^{1}$ School of Biotechnology, Jiangnan University, Wuxi, China \\ ${ }^{2}$ National Engineering Laboratory for Cereal Fermentation Technology, Jiangnan University, Wuxi, China \\ ${ }^{3}$ Affiliated Hospital of Jiangnan University, Department of Pediatrics, Wuxi, China \\ ${ }^{4}$ The Key Laboratory of Carbohydrate Chemistry and Biotechnology, Ministry of Education, School of Biotechnology, \\ Jiangnan University, Wuxi 214122, China
}

Correspondence should be addressed to Zhonghu Bai; baizhonghu@jiangnan.edu.cn and Xiao Tong; drtong@163.com

Received 8 February 2020; Revised 28 March 2020; Accepted 27 May 2020; Published 1 August 2020

Academic Editor: Guido Crisponi

Copyright (C) 2020 Shuang Han et al. This is an open access article distributed under the Creative Commons Attribution License, which permits unrestricted use, distribution, and reproduction in any medium, provided the original work is properly cited.

In this study, a chemiluminescence immunoassay (CLIA) for human serum 25-hydroxyvitamin D (25(OH)D) was established by a competition model. In serum, more than $99 \%$ of total circulating $25(\mathrm{OH}) \mathrm{D}$ binds to protein and less than $1 \%$ of $25(\mathrm{OH}) \mathrm{D}$ is in free form (Jassil et al., 2017). Before measuring concentration of 25(OH)D in serum, a releasing procedure should be conducted. A new reagent is used to release binding $25(\mathrm{OH}) \mathrm{D}$ to free form. Streptavidin (SA) was labeled to magnetic beads by a 1-ethyl-3-(3dimethylaminopropyl) carbodiimide/N-hydroxysuccinimide (EDC/NHS) method. Biotinylated VD was used as a competitor of $25(\mathrm{OH}) \mathrm{D}$ in samples. Anti-VD antibody (aby) was labeled to horseradish peroxidase (HRP) by EDC to react with $25(\mathrm{OH}) \mathrm{D}$ and biotinylated-VD molecules. The pretreated samples or standards were added into the reaction tube with biotin-VD and anti-VD aby-HRP, free $25(\mathrm{OH}) \mathrm{D}$ in the sample competes with biotinylated VD for binding to anti-VD aby-HRP, an SA-labeled magnetic particle is added to isolate the signal-generating complex, and the signal is inversely proportional to the $25(\mathrm{OH}) \mathrm{D}$ concentration in the sample. The method established shows good thermostability and performance. The limitation of detection (LoD) is $1.43 \mathrm{ng} /$ $\mathrm{mL}$. The intra-assay coefficient of variation $(\mathrm{CV})$ is $3.66 \%-6.56 \%$, the interassay $\mathrm{CV}$ is $4.19 \%-7.01 \%$, and the recovery rate is 93.22\%-107.99\%. Cross-reactivity (CR) was remarkably low with vitamin D2, vitamin D3, 1, 25-dihydroxyvitamin D3, and 1, 25dihydroxyvitamin D2. At the same time, the cross-reaction values with 25-hydroxyvitamin D2 and 25-hydroxyvitamin D3 were $97 \%$ and $100 \%$, respectively. The developed method shows good correlation with the total VD product from Roche and DiaSorin. 1096 clinical patient samples were measured with developed reagent kit in this study. 7 types of disease were involved, and the concentration of $25(\mathrm{OH}) \mathrm{D}$ is less than $30 \mathrm{ng} / \mathrm{mL}$ in $94.98 \%$ of patients.

\section{Introduction}

Vitamin D (VD), also known as sunshine vitamin, plays an important role in bone metabolism [1-3]. Vitamin D deficiency can cause growth retardation and skeletal deficiency in infant and children $[4,5]$, osteopenia, and osteoporosis usually happen in adult who have low-level vitamin $\mathrm{D}$ in circulation [6]. Vitamin D deficiency also has a possible role in chronic diseases, such as cancer [7], autoimmune diseases $[8,9]$, osteoarthritis [10], diabetes [11], and cardiovascular disease [12]. Therefore, detection of vitamin D concentration is a quite vital requirement of clinical diagnostics. 25(OH)D is the most widely used indicator of vitamin D status in either serum or plasma $[13,14]$. There are methods on the market for the analysis of $25(\mathrm{OH}) \mathrm{D}$. A radiological immunoassay was developed by SchiolerV in 1988 [15], which is time consuming and harmful to environment and operator's health. Several automated immunoassays were developed too, such as Liaison ${ }^{\circledR}$ Total Vitamin D, the IDS-iSYS 25-Hydroxy Vitamin D, the ARCHITECT 25-OH Vitamin $\mathrm{D}$, and the ADVIA Centaur ${ }^{\circledR}$ Vitamin D Total, and none of these immunoassays gave results equivalent to the liquid chromatography-tandem mass spectrometry (LC-MS/MS) method [16]. High-performance liquid chromatography 
spectrometry was developed for 25(OH)D detection [17], but this method is quite expensive and requires special training for the operator. In contrast, CLIA is a simple, sensitive, and cheap method for the high-throughput quantification of analyses in samples. In this study, a direct competitive immunoassay was established on the CLIA platform.

\section{Materials and Methods}

2.1. Reagents and Materials. Dynabeads MyOne ${ }^{\mathrm{TM}}$ carboxylic acid beads, EZLink ${ }^{\mathrm{TM}}$ Sulfo-NHS-LC-Biotinylation Kit, succinimidyl 4-(N-maleimidomethyl)cyclohexane-1-carboxylate (SMCC), and 4'-hydroxyazobenzene-2-carboxylic acid (HABA) solution are obtained from Thermo Fisher; perfluorohexanoate(PFHxA), methanol, EDC, and NHS are purchased from Sigma; an AKTA purifier is purchased from GE healthcare; biotinylated vitamin D (BVD) is obtained from DIASource; HRP is purchased from BBI solutions; streptavidin is purchased from NeuroPeptide from China; a microscope is purchased from Olympus; an automicroplate chemiluminescent analyzer is supplied by Baiming Biotechnology from China; and an automagnetic beads chemiluminescent analyzer is supplied by Zecheng Biotechnology from China. Mice used for antibody production are obtained from Jiangnan University; VD is purchased from Conju-Probe.

\subsection{Antibody Immunization and Purification}

2.2.1. Antibody Immunization. 25-hydroxyvitamin D was conjugated to bovine serum albumin (BSA) by a SMCC crosslinker before immunization. Three female BALB/c mice were used as hosts, and each mouse weighed $20 \mathrm{~g}$ and was 4 weeks old. Hosts were immunized 3 times with 50 ug VDBSA in complete Freund's adjuvant mixture, and the immunization interval was 3 weeks, at a last boost with $100 \mathrm{ug}$ VD-BSA in incomplete Freund's adjuvant. Splenocytes were harvested from immunized mice in one week, then fusion with SP2/0 cells.

2.2.2. Antibody Screening and Purification. ELISA assay was employed to screen target anti-VD antibody. Biotinylated $25(\mathrm{OH}) \mathrm{D}$ was used as a probe. HRP-conjugated anti-mouse antibody was used to generate signals. 96-well plates were coated with streptavidin (SA) as solid phase. One primary screening from the supernatant of splenocyte-SP $2 / 0$ fusion cells was performed. 3 rounds of subcloning were conducted to obtain hybridoma-secreting anti-VD monoclonal antibody. The anti-VD monoclonal antibodies were further validated as raw material to build $25(\mathrm{OH}) \mathrm{D}$ detection reagent kit.

\subsection{Reagent Component Preparation}

2.3.1. Streptavidin-Coated Magnetic Bead Preparation [18]. Dynabeads MyOne was washed twice with $25 \mathrm{mM}$ 2-morpholinoethanesulfonic acid (MES) buffer, $\mathrm{pH} 6.0$, to remove storage buffer of the particle. EDC and NHS solutions were added to the Dynabeads to active the binding group on particle surface, suspended the particle and well mixed all compounds. The mixture was incubated with gently tilt rotation at room temperature for 30 minutes. The beads were washed two more times to remove the supernatant. The required amount of streptavidin was added into preactivated magnetic beads and incubated for another 30 minutes at room temperature with gently tilt rotation. At last, the particle was washed twice again and then suspended to storage concentration with PBS buffer containing 0.5\% BSA, $0.05 \%$ polysorbate- 20 , and $0.02 \%$ sodium azide.

\subsubsection{Characterization of SA-Coated Magnetic Particle.} The biotin-streptavidin system is used to test quality of the in-house prepared magnetic particle. Two sets of reaction tubes are prepared, $30 \mu \mathrm{L}$ in-house prepared SA-coated magnetic particle is added, and $50 \mu \mathrm{L}$ biotinylated HRP $(50 \mathrm{ng} / \mathrm{mL})$ is added to one set and $50 \mu \mathrm{L} \mathrm{HRP}(50 \mathrm{ng} / \mathrm{mL})$ is added to the other set. Both sets were incubated for $30 \mathrm{~min}$ at $37^{\circ} \mathrm{C}$, and then the particles were washed 3 times with washing buffer. At last, $100 \mu \mathrm{L}$ substrate reagent is added to generate signals. The RLUs are 710,325 with biotinylated HRP and 352 with HRP. This result indicated that the inhouse prepared magnetic particle is ready to use (see Supplementary Table 1).

2.3.3. Antibody Conjugates with HRP. Different weights of anti-VD antibody and EDC are dissolved in separated $0.05 \mathrm{M}$ sodium bicarbonate solutions. Mix them after the solute dissolved completely. HRP is dissolved in 0.1 Mphosphate buffer, $\mathrm{pH}$ 7.2. Then, the mixed solution is added into the HRP solution and the $\mathrm{pH}$ is lowered to 5.8 with diluted hydrochloric acid. It is incubated for 5 hours at room temperature. Finally, the impurities are removed with a desalination column.

2.3.4. Characterization of Anti-VD Antibody-HRP Conjugates. The anti-VD antibody-HRP conjugate is prepared in different antibody/HRP ratios. Biotin-VD-SA magnetic particle coupling is used as a probe to test quality of the anti-VD antibody-HRP conjugate. Several sets of reaction tubes are prepared, $30 \mu \mathrm{L}$ biotin-VD-SA magnetic particle coupling is added, and $50 \mu \mathrm{L}$ different anti-VD antibody-HRP is added with HRP as control into reaction tubes, then incubated for $30 \mathrm{~min}$ at $37^{\circ} \mathrm{C}$; the particle is washed 5 times with washing buffer. Then, $100 \mu \mathrm{L}$ substrate reagent is added. The results indicate that anti-VD antibodyHRP conjugates are ready to use (see Supplementary Figure 1).

2.4. Reaction Condition Optimization. In serum, more than $99 \%$ of total circulating VD binds to protein and less than $1 \%$ of VD is in free form. A VD-releasing process should be involved before measuring the concentration of total VD in serum. Alkaline condition and DTT are used by Roche. In this study, a new reagent has been developed to releasing VD 
which is more efficiency and time-saving; PFHxA and methanol were used as key material in a PH 7.5 buffer. The essential reagents required for this assay include biotin-VD, anti-VD antibody-HRP conjugates, and VD. Upon mixing the anti-VD antibody-HRP conjugates with a serum pretreated containing free $\mathrm{VD}$, a reaction results between the VD and anti-VD antibody. Then, the magnetic particle with binding biotinylated VD was added, competed binding to the remnant anti-VD antibody-HRP conjugate. The RLUs generated are inversely proportional to the VD concentration. By utilizing several different serum references of known VD concentration, a concentration response curve can be generated, and the VD concentration of an unknown can be ascertained. In this study, factors such as VD-releasing procedure, biotin-VD-magnetic particle concentration, antiVD antibody-HRP type and concentration, and incubation time were optimized.

2.4.1. Method Procedure. We added $30 \mu \mathrm{L}$ serum and $150 \mu \mathrm{L}$ VD-releasing reagent into a reacting tube, mixed, and incubated for 18 minutes at $37^{\circ} \mathrm{C}$ to release VD from binding protein. $50 \mu \mathrm{L}$ anti-VD antibody-HRP is added into a reacting tube and incubated for 15 minutes at $37^{\circ} \mathrm{C}$, then added $30 \mu \mathrm{L}$ magnetic particle coated with biotinylated VD and incubated for another $5 \mathrm{~min}$ at $37^{\circ} \mathrm{C}$. After incubation, the magnetic particle was washed three times with washing buffer, and then $200 \mu \mathrm{L}$ substrate reagent is added to generate signals. A signal reader is used to collect RLUs, and concentration values are calculated if the calibrator curve is available.

2.4.2. Optimization of Biotin-VD and Anti-VD AntibodyHRP Concentration. Different antibody/HRP ratio conjugates present different competing abilities. Signals of standards increase along with the excess molar increase of HRP. The signal $(0 \mathrm{ng} / \mathrm{mL}) /$ signal $(150 \mathrm{ng} / \mathrm{mL})$ value increases first and then remains at the same level, and the largest number is under condition of antibody/HRP ratio at $2: 1$. The results are shown in Supplementary Figure 2. A series concentration of biotinylated VD and anti-VD antibody-HRP was investigated, and the signals and signal $(0 \mathrm{ng} / \mathrm{mL}) /$ signal $(150 \mathrm{ng} /$ $\mathrm{mL}$ ) value are acceptable when biotin-VD concentration is $30 \mathrm{ng} / \mathrm{mL}$ and anti-VD antibody-HRP concentration is $1,000 \mathrm{ng} / \mathrm{mL}$. The results of optimization are shown in Supplementary Table 2.

2.4.3. Optimization of Incubation Time. The biotinylated VD and anti-VD antibody-HRP incubation time is studied, respectively. The procedure remains unchanged described in the method procedure except for the incubation time. For biotinylated VD, signals remain the same in our test time point. For anti-VD antibody-HRP, signals increase as the incubation time is lengthened at first but remain constant while the reaction achieves dynamic equilibrium. Due to time-saving requirement of clinical, we choose $5 \mathrm{~min}$ incubation of biotinylated VD and 16 min incubation of antiVD antibody-HRP as the best incubation condition which achieves high signals with a relative low variable coefficient. The signal results are shown in Supplementary Figure 3 and Table 3.

2.4.4. VD-Releasing Procedure Optimization. PFHxA and methanol were used as key material to release the binding $25(\mathrm{OH}) \mathrm{D}$ to free form. PFHxA is used as a main releasing compound while methanol as a cosolvent, with a neutral $\mathrm{PH}$, and the releasing reagent has low effect of following reactions. Theoretically, the more the PFHxA is added, the more efficient the releasing reagent will be; however, according to results with more than $1 \% \mathrm{PFHxA}$ in the reagent, the final RLU was decreased. Therefore, 1\% PFHxA was the chosen concentration. Data are shown in Supplementary Figure 4 . The concentration of methanol was optimized, the CV value became better with methanol in the releasing buffer, $5 \%$ was the final concentration, and data are shown in Supplementary Figure 5. Finally, the releasing time of $25(\mathrm{OH}) \mathrm{D}$ was optimized, data are shown in Supplementary Figure 6. The concentrations of samples hardly increase after $18 \mathrm{mins}$, so it is chosen as the final releasing time.

\subsection{Performance Test Method}

2.5.1. Limitation of Detection. 20 standards with $0 \mathrm{ng} / \mathrm{mL}$ analyte are tested, and then MEAN-3SD value is calculated. The corresponding concentration is the limitation of detection.

2.5.2. Precision and Recovery. Three serum samples of different $25(\mathrm{OH}) \mathrm{D}$ concentrations were tested and duplicated separately in one experiment and repeated in 20 days, and the intra- and interassay CV are calculated. The concentration of $25(\mathrm{OH}) \mathrm{D}$ solutions was added to three serum samples with different analyte levels, and the recovery rate is calculated.

2.5.3. Cross-Reactivity. The specificity of the anti-VD antibody used to selected substances was evaluated by adding the interfering substance to a serum matrix at various concentrations. The cross-reactivity (CR) is defined at the point where the reduction in the signal corresponds to $50 \%$ of the signal achieved in the absence of analyte (B/B0 of $50 \%$ ), as a percentage of the analyte concentration given the same fall in the signal. The $\mathrm{CR}$ values were calculated as follows: $\mathrm{CR}(\%)=$ IC50 of $25(\mathrm{OH}) \mathrm{D} / \mathrm{IC} 50$ of competitor $\times 100 \%$.

2.5.4. Accelerated Stability. The whole kit including biotinylated VD, SA-coated magnetic particle, anti-VD antibody-HRP, and VD standards was incubated at $37^{\circ} \mathrm{C}$ for 7 days, and the signals of standards and samples at different days were compared.

2.5.5. Method Comparison. The established method was compared with two on-market CLIA methods from Roche 

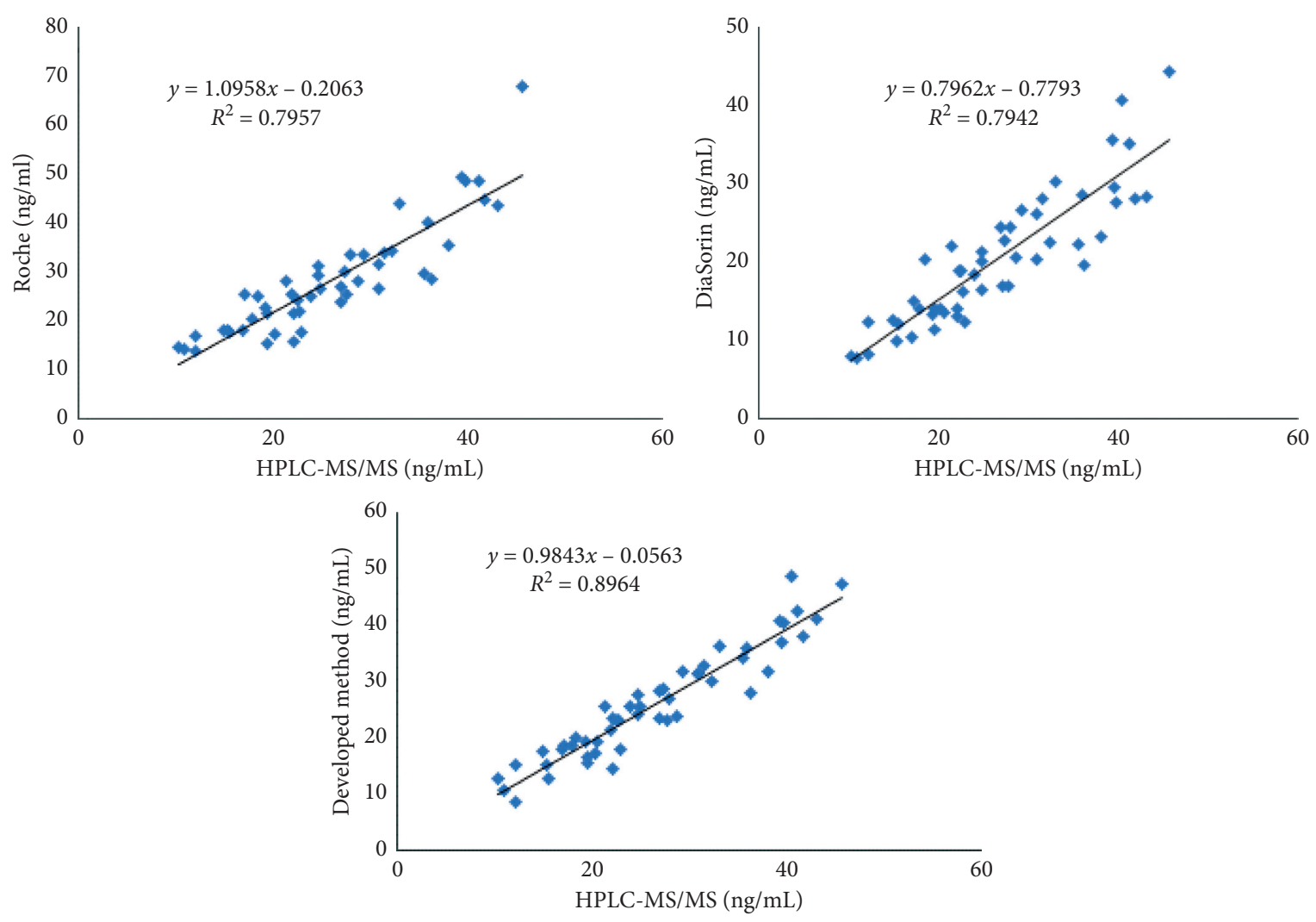

FIgURE 1: Comparison with UPLC-MS/MS.

Table 1: Precision result.

\begin{tabular}{lcccccc}
\hline \multirow{3}{*}{ Samples } & \multicolumn{3}{c}{ Intra-assay CV $(n=20)$} & \multicolumn{2}{c}{ Interassay CV $(n=20)$} \\
& Mean (ng/mL) & SD & $\begin{array}{c}\text { CV } \\
(\%)\end{array}$ & $\begin{array}{c}\text { Mean }(n g / \\
\text { mL) }\end{array}$ & SD & $\begin{array}{c}\text { CV } \\
(\%)\end{array}$ \\
\hline 1 & 9.91 & 0.65 & 6.56 & 9.56 & 0.67 & 7.01 \\
\hline 2 & 24.93 & 1.13 & 4.53 & 24.37 & 1.02 & 4.19 \\
3 & 50.52 & 1.85 & 3.66 & 50.18 & 2.14 & 4.26 \\
\hline
\end{tabular}

and DiaSorin. The sample size used to compare with Roche is 244 and ranged from $3 \mathrm{ng} / \mathrm{ml}$ to $63.88 \mathrm{ng} / \mathrm{ml}$. 202 serum samples are used to compare with DiaSorin with range from $6.64 \mathrm{ng} / \mathrm{ml}$ to $89.00 \mathrm{ng} / \mathrm{ml}$.

\section{Result}

3.1. VD-Releasing Reagent Efficiency Test. Efficiency of the releasing reagent was tested by comparing test results with HPLC-MS/MS concentration. The correlation equation of the developed method with HPLC-MS/MS is $y=0.984 \mathrm{x}-0.056$, and correlation coefficient is 0.9468 . Results are shown in Figure 1. At the same time, samples were tested on the DiaSorin and Roche platforms side by side. The correlation of the DiaSorin method with HPLC-MS/MS is $y=0.796 x-0.779$, and correlation coefficient is 0.8911 . The correlation of the Roche method with HPLC-MS/MS is $y=1.095 x-0.206$, and correlation coefficient is 0.8920 .
TABLE 2: Analytical recovery.

\begin{tabular}{ccccc}
\hline \multirow{2}{*}{ Samples } & \multicolumn{4}{c}{ VD concentration $(\mathrm{ng} / \mathrm{mL})$} \\
& Added concentration & Tested & Expected & Recovery $(\%)$ \\
\hline \multirow{4}{*}{1} & 0.00 & 9.76 & 10.54 & 107.99 \\
& 5.63 & 15.88 & 15.48 & 97.48 \\
& 11.25 & 20.98 & 19.90 & 94.85 \\
& 22.50 & 31.18 & 31.97 & 102.53 \\
& 45.00 & 58.99 & 54.99 & 93.22 \\
\hline & 0 & 23.53 & 24.56 & 104.38 \\
& 5.625 & 29.59 & 29.21 & 98.72 \\
& 11.25 & 35.10 & 34.87 & 99.34 \\
& 22.5 & 47.20 & 45.76 & 96.95 \\
& 45 & 65.29 & 67.90 & 104.00 \\
\hline
\end{tabular}

3.2. Method Performance. The 4-parameter logic function method was used to fit the standard curve of signal with $25(\mathrm{OH}) \mathrm{D}$ concentration. The following series concentrations of $25(\mathrm{OH}) \mathrm{D}$ were used as a standard curve, $0 \mathrm{ng} / \mathrm{mL}$, $10 \mathrm{ng} / \mathrm{mL}, 25 \mathrm{ng} / \mathrm{mL}, 50 \mathrm{ng} / \mathrm{mL}, 90 \mathrm{ng} / \mathrm{mL}$, and $150 \mathrm{ng} / \mathrm{mL}$. The LoD is $1.43 \mathrm{ng} / \mathrm{ml}$. Precision was tested, intra-assay CV $\%$ is $3.66 \%-6.56 \%$, interassay CV \% is $4.19 \%-7.01 \%$, and the results are shown in Table 1 . The recovery rate is $93.22 \%-$ $107.99 \%$, and the results are shown in Table 2. The developed methods have high selectivity for $25(\mathrm{OH}) \mathrm{D}$. Cross-reaction values were less than $0.1 \%$ with vitamin $\mathrm{D} 2$, vitamin $\mathrm{D} 3,1$, 25-dihydroxyvitamin D3, and 1, 25-dihydroxyvitamin D2. At the same time, the cross-reaction values with 25hydroxyvitamin D2 and 25-hydroxyvitamin D3 were 97\% 


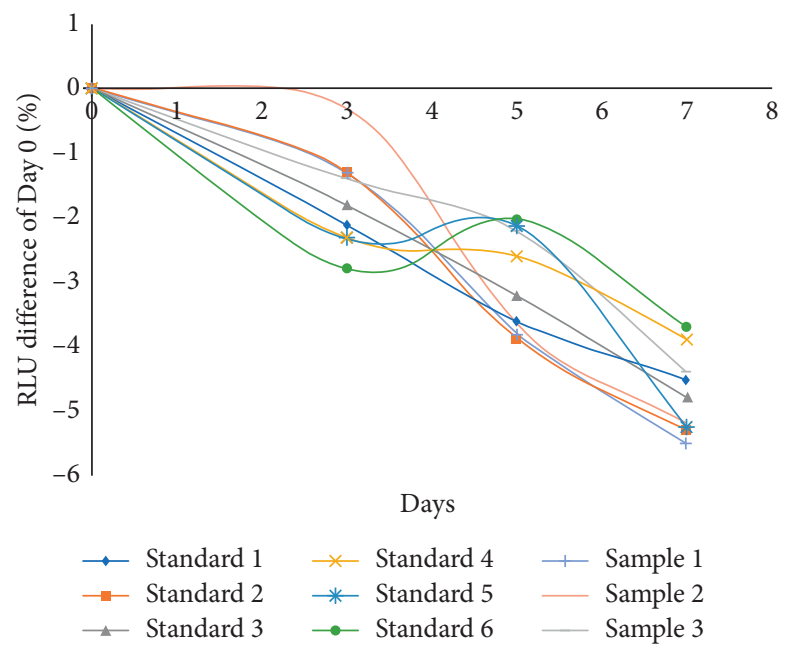

FIGURE 2: Reagent kit accelerated stability.
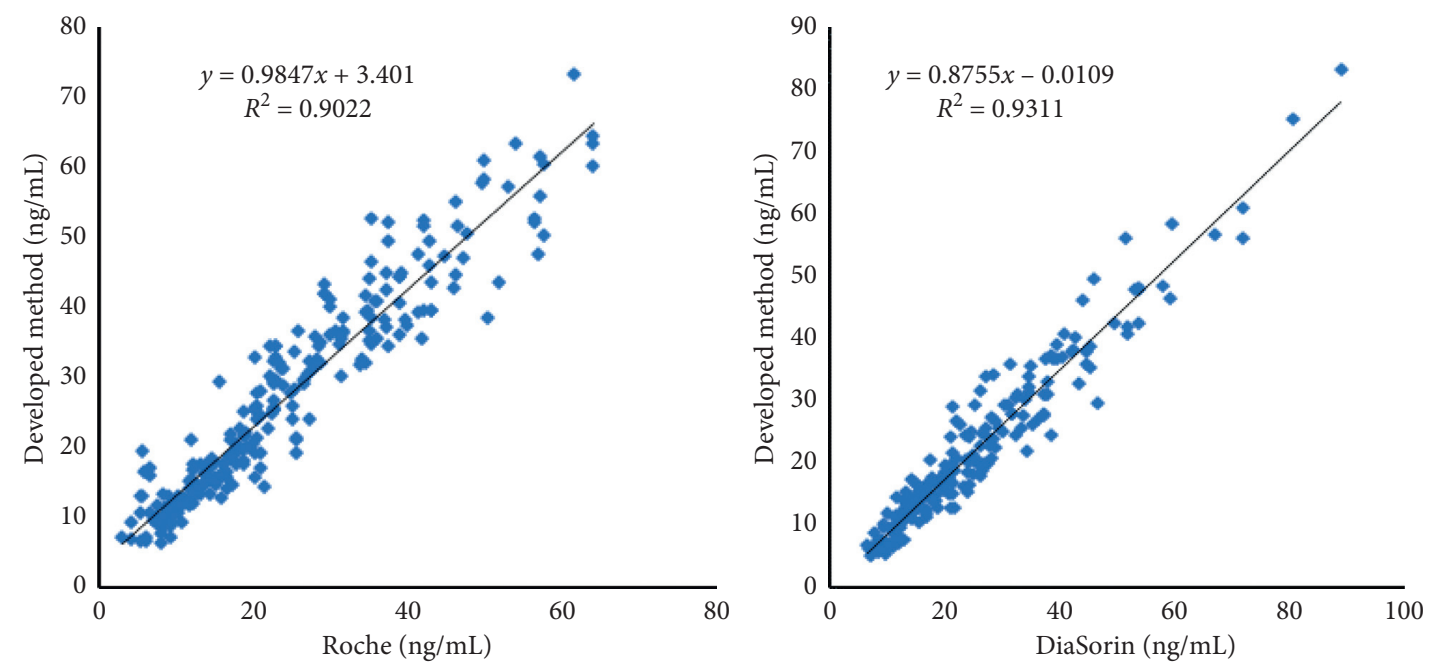

FIgURE 3: Comparison with on-market products.

TABLE 3: Distribution of clinical samples.

\begin{tabular}{lccccccc}
\hline Conc. of 25(OH)D & Fracture & Osteoporosis & Osteoarthritis & Autoimmune diseases & Cancer & Diabetes & Cardiovascular disease \\
\hline$<30 \mathrm{ng} / \mathrm{mL}$ & 41 & 25 & 55 & 140 & 203 & 524 & 53 \\
$>30 \mathrm{ng} / \mathrm{mL}$ & 0 & 4 & 1 & 16 & 6 & 27 & 1 \\
Total & 41 & 29 & 56 & 156 & 209 & 551 & 54 \\
\hline
\end{tabular}

and $100 \%$, respectively. Accelerated stability study was performed; the developed reagent showed great stability under stressed temperature. The RLUs changed less than $10 \%$ during 7 -day periods under $37^{\circ} \mathrm{C}$, see the results in Figure 2.

3.3. Method Comparison. In this study, 244 serum samples were measured by both the developed and Roche methods and 202 serum samples were measured by both the developed and DiaSorin methods. The test results were regressed by the least square regression equation, and the correlation coefficient was computed too. Data are shown in Figure 3.
Test results show good agreement between the developed method and compared methods, and difference of the test values and mean values indicated slight amounts of bias between developed and compared methods.

3.4. Clinical Study. 1096 clinical patient samples were measured with developed reagent kit in this study. Samples were treated using the same method we described in Section 2.4.4. 7 types of disease were involved, and the concentration of $25(\mathrm{OH}) \mathrm{D}$ is less than $30 \mathrm{ng} / \mathrm{mL}$ in $94.98 \%$ patients. Data are shown in Table 3. Among patients have low-level 25(OH) D, nearly $50 \%$ are related to diabetes, about $20 \%$ patients 


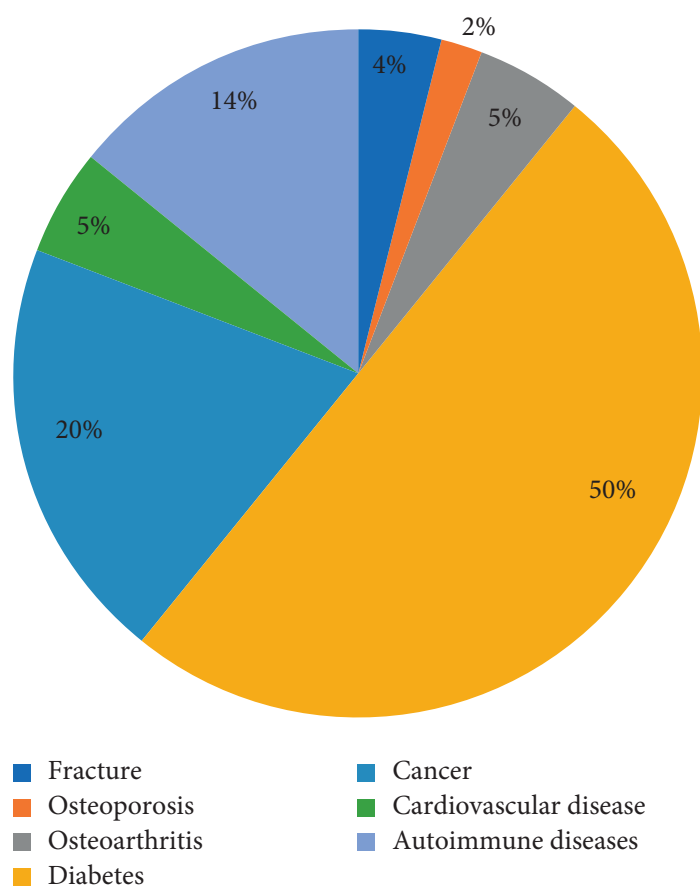

Figure 4: Distribution of clinical samples that $25(\mathrm{OH}) \mathrm{D}$ is less than $30 \mathrm{ng} / \mathrm{mL}$.

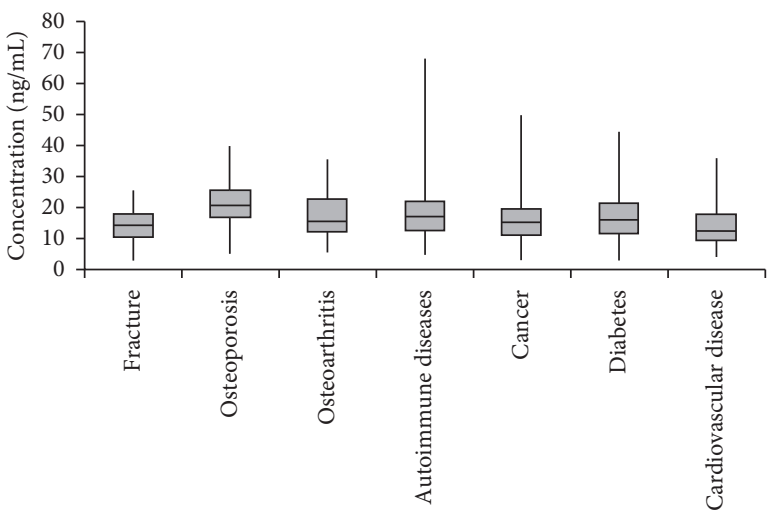

FIGURE 5: Concentration of 25(OH)D of different patients.

have cancers, and almost $14 \%$ got fracture. Data are shown in Figure 4. Patients who have autoimmune disease, cancer, and diabetes have wider range of $25(\mathrm{OH}) \mathrm{D}$ level, patients who got fracture, osteoporosis, and osteoarthritis have narrow range of $25(\mathrm{OH}) \mathrm{D}$, and almost all are lower than $30 \mathrm{ng} / \mathrm{mL}$. Data are shown in Figure 5.

\section{Discussion and Conclusion}

According to the previous literature studies, there are many quantification methods for $25(\mathrm{OH}) \mathrm{D}$, such as antibodybased isoluminol derivate direct competitive two-step chemiluminescent system used by DiaSorin; antibody-based acridinium ester competitive assay used by IDS plc, Abbott, and Siemens; 25-OH-vitamin D binding protein based electrochemiluminescence competitive assay used by Roche; and LC-MS/MS-based nonimmunological direct detection method used by PerkinElmer. Due to the differences of the sample pretreatment method and specificity of biomaterial employed in different immunoassays, the agreement of test result is not very good. LC-MS/MS is usually considered as a reference method, but the cost of instruments, consumables, and the low throughput limited its wide application. This study established a chemiluminescence immunoassay for the quantification of $25(\mathrm{OH}) \mathrm{D}$ in human serum with good performance and overall stability. The method comparison result shows that this method has a good correlation with the 25(OH)D kit from Roche and DiaSorin which are highly admitted and widely used on the market. Samples results compared with HPLC-MS/MS in releasing reagent optimization section show that this method has a good agreement with the physical detection method which usually be considered as a reference method. In serum, 25(OH)D binds to protein as a complex; pretreatment of the sample should be performed before the test. In this study, a new releasing reagent was employed to maximize the release of $25(\mathrm{OH}) \mathrm{D}$; besides, the $\mathrm{PH}$ of the releasing reagent is around 7.5 which has low effect on following reactions. In this study, in-house produced biomaterial such as SA-coated magnetic particle, anti-VD antibody, and anti-VD antibody-HRP conjugate are critical to the reagent performance. Therefore, further studies should be conducted on the antibody-producing procedure and coupling conjugate procedure. Minimization of batch difference of raw material should help to sustain performance of reagent kit. The clinical study with developed kits shows good agreement of the test result and disease, and the reagent kit was fulfilling for the clinical requirement.

\section{Data Availability}

The data used to support the findings of this study are available from the corresponding author upon request.

\section{Conflicts of Interest}

The authors declare that they have no conflicts of interest.

\section{Authors' Contributions}

Shuang Han and Wuxian Qiu equally contributed to this work.

\section{Acknowledgments}

This work was supported by Jiangsu Key R \& D Program (BE2018621), Postgraduate Research Innovation Program of Jiangsu Provence (KYCX18_1793), National First-Class Discipline Program of Light Industry Technology and Engineering (LITE2018-24), and the Fifteenth Batch of the "Six Talent Peaks Project in Jiangsu Province” (SWYY-180).

\section{Supplementary Materials}

Figure S1: characterization of anti-VD antibody-HRP conjugate $(n=3)$. Figure S2: performance of different 
antibody/HRP ratio conjugates. Figure S3: optimization of incubation time. Figure S4: optimization of PFHxA concentration. Figure S5: optimization of methanol concentration. Figure S6: optimization of VD-releasing time. Table S1: characterization of the magnetic particle. Table S2: biotin-VD and anti-VD antibody-HRP concentration optimization. Table S3: precision results of optimization incubation time. (Supplementary Materials)

\section{References}

[1] N. K. Jassil, A. Sharma, D. Bikle, and X. Wang, "Vitamin D binding protein and 25-hydroxyvitamin D levels: emerging clinical applications," Endocrine Practice, vol. 23, no. 5, pp. 605-613, 2017.

[2] P. H. Anderson, "Vitamin D activity and metabolism in bone," Current Osteoporosis Reports, vol. 15, no. 5, pp. 443-449, 2017.

[3] P. Lips and N. M. van Schoor, "The effect of vitamin D on bone and osteoporosis," Best Practice \& Research Clinical Endocrinology \& Metabolism, vol. 25, no. 4, pp. 585-591, 2011.

[4] B. W. Hollis and C. L. Wagner, "Assessment of dietary vitamin D requirements during pregnancy and lactation," The American Journal of Clinical Nutrition, vol. 79, no. 5, pp. 717-726, 2004.

[5] B. W. Hollis and C. L. Wagner, "Vitamin D requirements during lactation: high-dose maternal supplementation as therapy to prevent hypovitaminosis D for both the mother and the nursing infant," The American Journal of Clinical Nutrition, vol. 80, no. 6, pp. 1752S-1758S, 2004.

[6] F. Michael and M. D. Holick, "Vitamin D deficiency," New England Journal of Medicine, vol. 357, no. 3, pp. 266-281, 2007.

[7] A. M. Mondul, S. J. Weinstein, T. M. Layne, and D. Albanes, "Vitamin D and cancer risk and mortality: state of the science, gaps, and challenges," Epidemiologic Reviews, vol. 39, no. 1, pp. 28-48, 2017.

[8] YH. Lee and S. C. Bae, "Vitamin D level in rheumatoid arthritis and its correlation with the disease activity: a metaanalysis," Clinical and Experimental Rheumatology, vol. 34, no. 5, pp. 827-833, 2016.

[9] D. S. Smyk, T. Orfanidou, P. Invernizzi, D. P. Bogdanos, and M. Lenzi, "Vitamin D in autoimmune liver disease," Clinics and Research in Hepatology and Gastroenterology, vol. 37, no. 5, pp. 535-545, 2013.

[10] P. Manoy, P. Yuktanandana, A. Tanavalee et al., "Vitamin D supplementation improves quality of life and physical performance in osteoarthritis patients," Nutrients, vol. 9, no. 8, p. E799, 2017.

[11] S. J. Wimalawansa, "Associations of vitamin D with insulin resistance, obesity, type 2 diabetes, and metabolic syndrome," The Journal of Steroid Biochemistry and Molecular Biology, vol. 175, pp. 177-189, 2018.

[12] R. Scragg, A. W. Stewart, D. Waayer et al., "Effect of monthly high-dose vitamin D supplementation on cardiovascular disease in the vitamin D assessment study," JAMA Cardiology, vol. 2, no. 6, pp. 608-616, 2017.

[13] B. W. Hollis, "Measuring 25-hydroxyvitamin D in a clinical environment: challenges and needs," The American Journal of Clinical Nutrition, vol. 88, no. 2, pp. 507S-510S, 2008.

[14] M. F. holick, "Vitamin D status: measurement, interpretation, and clinical application," Annals of Epidemiology, vol. 19, no. 2, pp. 73-78, 2009.
[15] T.J. SchiolerV, "Six direct radioimmunoassay of estradiol evaluated," Clinical Chemistry, vol. 34, no. 5, pp. 949-952, 1988.

[16] M.-k. Koivula, N. Matnlassi, and J. R. Paivilaitinen, "Four automated $25-\mathrm{OH}$ total vitamin D immunoassays and commercial liquid chromatography tandem-mass spectrometry in Finnish population," Clinical Laboratory, vol. 59, no. 3-4, pp. 397-405, 2013.

[17] J. Plíšek, L. K. Krčmová, J. Aufartová et al., "New approach for the clinical monitoring of 25-hydroxyvitamin D3 and 25hydroxyvitamin D2 by ultra high performance liquid chromatography with MS/MS based on the standard reference material 972," Journal of Seperation Science, vol. 36, no. 23, pp. 3702-3708, 2013.

[18] X. Chen, Q. Zhou, T. Zhang et al., "Development of a sensitive chemiluminescence immunoassay for the quantification of folic acid in human serum," Journal of Analytical Methods in Chemistry, vol. 2019, Article ID 5402903, 7 pages, 2017. 